\title{
LA CONSTRUCCIÓN DE LOS IRRACIONALES DE DEDEKIND COMO INSTRUMENTO EN UN ANÁLISIS DE TEXTOS DE OCTAVO GRADO
}

\author{
Jeannette Vargas Hernández \\ Magíster en Docencia de la Matemática. Universidad Pedagógica Nacional. \\ Docente Universidad Colegio Mayor de Cundinamarca \\ yanow43@hotmail.com
}

\section{RESUMEN}

El continuo numérico, hace parte de los conceptos del currículo en la básica secundaria, y es tanto la complejidad que conlleva su enseñanza y su aprendizaje como la incidencia que tienen los textos escolares en dichos procesos, lo que nos lleva a delimitar esta investigación como un análisis de textos. Para ello se expone el contexto de la investigación tomando como base un segmento del desarrollo histórico del mismo; puntualizando en los aportes realizados por Richard Dedekind en la formalización de este concepto.

Palabras claves: Continuo numérico, análisis, textos, escolar, educación, infinito, actual, potencial, número, irracional, real, recta, punto, cortadura, orden, completez, construcción.

\section{SUMMARY}

The continuous numeric, makes part of the concepts of the curriculum in the basic secondary, and it is so much the complexity that bears their teaching and their learning like the incidence that have the school texts in this processes, what takes us to define this investigation like an analysis of texts. For it is exposed it the context of the investigation taking like base a segment of the historical development of the same one; remarking in the contributions carried out by Richard Dedekind in the formalization of this concept.

Keywords: Continuous numeric, analysis, texts, school, education, infinite, current, potential, number, irrational, real, straight line, point, cut, order, completez, construction.

\section{CONTEXTO DE LA INVESTIGACIÓN}

En el sistema escolar colombiano; en el currículo, el tema de los números reales está designado para ser enseñado, lo que obliga a tener en cuenta las múltiples implicaciones que dicha decisión conlleva para los diferentes agentes que intervienen en los procesos de enseñanza y aprendizaje.

Desde la práctica docente se observa que el paso de los números racionales a los reales resulta complejo tanto para estudiantes como para maestros. El docente, en determinados momentos de manera consciente y en otros de manera mecánica, presenta este paso sin permitir que el estudiante establezca diferencias conceptuales profundas entre el dominio numérico de los racionales y el de los reales. 
En la aproximación a la conceptualización, los textos son una herramienta de apoyo, tanto para docentes como para estudiantes en los procesos de enseñanza y aprendizaje. La importancia del texto se puede describir en qué "impone una distribución y una jerarquía de los conocimientos y contribuye a forjar los andamios intelectuales tanto de los maestros como de los alumnos", citado por García, Serrano y Espitia.

\section{DELIMITACIÓN DEL PROBLEMA}

Con el fin de delimitar el problema de investigación se plantea la pregunta: ¿De qué manera se presenta el continuo numérico en los textos escolares de matemáticas?

Para acercarse a una respuesta, se centra el estudio en:

- Describir y caracterizar la forma como se presentan los números naturales, enteros y racionales.

- Describir y caracterizar la forma como se presentan los números irracionales y reales.

- Determinar la presencia del infinito potencial y del infinito actual.

- Analizar la presentación del continuo numérico en los textos escolares.

Descripción: Investigación descriptiva, cualitativa e interpretativa, que consiste en la elaboración, de un análisis de textos con el fin de responder a la pregunta, ¿De qué manera se presenta el continuo numérico en los textos escolares de matemáticas?

Metodología: Al determinar el contexto de investigación dentro del ambiente escolar, se ubican dos espacios, uno de ellos, los conceptos a ser enseñados y el otro, las herramientas de apoyo para los procesos de aprendizaje de dichos conceptos.

En lo referente a los conceptos se escogió el continuo numérico y en relación con las herramientas de apoyo, los textos escolares de matemáticas de octavo grado. En relación con el continuo numérico, se plantearon las preguntas: ¿Qué es un número? ¿Qué es número real? ¿En qué consiste la continuidad? y, ¿en qué sentido el paso del infinito potencial al infinito actual garantiza el paso de los racionales a los reales, o de la enumerabilidad a la continuidad? Preguntas que nos llevaron a consultar bibliografía especializada en el tema con el fin de construir el marco teórico para esta investigación.

En la selección de los textos se utilizó como criterio la identificación de los textos más usados en octavo grado en el área de matemáticas, y, para analizar de qué manera se presenta el continuo numérico en los textos escolares, se establecieron categorías de análisis, tomando como base los referentes teóricos. Teniendo en cuenta los elementos definidos, se elaboró un análisis de textos y se plantearon algunas conclusiones y sugerencias.

Es importante anotar que este análisis de textos no se ciñó a ningún esquema establecido, para tal fin, optamos por utilizar algunos aspectos enunciados por diferentes autores y establecimos un esquema propio, dado que se considero que esta construcción no constriñe la mirada hacia los objetos de estudio. 


\section{REFERENTES TEÓRICOS}

Un aspecto teórico de la historia de la matemática se abordó a través de la pregunta: ¿Qué es número real?, encontrando documentación en donde se afirma que se llega al siglo XIX con el siguiente concepto de número: número racional es el que puede representarse por medio de una expresión decimal periódica y número irracional el que no tiene un período en su expresión decimal. Pero se hacía necesario dar una definición de número irracional independiente del sistema de numeración y se mencionan cuatro importantes trabajos sobre dicha construcción: (a) Karl Weierstrass (1815-1897) (b) Charles Méray (1835-1911), (c) George Cantor (1845-1918), (d) Richard Dedekind (1831-1916).

De las construcciones, en esta investigación se centra la atención en la construcción realizada por Dedekind, dado que en ésta se aborda de manera explícita la continuidad de los reales; con el fin de profundizar un poco más en esta construcción nos remitimos al libro “¿Qué son y para qué sirven los números?”, y de los capítulos: La construcción del sistema numérico, y Continuidad y números irracionales, se presenta a continuación unos párrafos de la síntesis.

Dedekind se expresa de la siguiente manera: "Cada punto $p$ de la recta produce una separación de la misma en dos porciones de manera que cada punto de una parte está a la izquierda de cada punto de la otra parte. Encontré la esencia de la continuidad en el recíproco, es decir en el siguiente principio: Si todos los puntos de la línea recta son de dos clases, de manera que cada punto de la primera clase está a la izquierda de cada punto de la segunda clase, entonces existe un punto y únicamente uno que ocasiona la partición de todos los puntos en dos clases separando la recta en dos partes... La suposición de esta propiedad de la recta no es más que un axioma por el cual atribuimos a la línea por primera vez su continuidad." p. 85

A continuación se afirma en el texto que si se desea seguir aritméticamente todos los fenómenos de la recta , los números racionales no bastan para ello, y que en consecuencia se hace necesario crear nuevos números. Dedekind plantea entonces que el dominio discontinuo de los números racionales puede rehacerse hasta formar un dominio continuo, para lo cual define las cortaduras y plantea que hay infinidad de cortaduras que no son producidas por números racionales. Cada cortadura no producida por un número racional produce un nuevo número, llamado número irracional.

Define los reales y encamina su trabajo a demostrar que el dominio de los reales posee continuidad, es decir que se cumple el siguiente teorema: Si el sistema R de todos los números reales se divide en dos clases $U_{1}$ y $U_{2}$ tales que cada número $a_{1}$ de la clase $U_{1}$ es menor que cada número $a_{2}$ de la clase $U_{2}$, entonces existe un solo número a que puede producir dicha separación.

Pero no es únicamente el concepto de continuidad el que se encuentra en el libro ¿Qué son y para qué sirven los números?, es la estructura que se describe en el libro, sobre sus investigaciones y los elementos que retomó Dedekind en algunos de sus estudios, lo que apoya las categorías de análisis de los textos. Es decir, la lectura detallada nos permitió extraer elementos y, tomar la esencia o las huellas que se vislumbran en su trabajo. Estas son algunas frases que se extractan del libro: 
“... basta saber que esa limitación en la posibilidad de realizar las operaciones indirectas es precisamente, en cada caso, la verdadera causa de un nuevo acto creativo; así los números negativos y quebrados han sido creados por la mente humana..."

"...la introducción de los números irracionales habitual hasta el momento se refiere directamente a la noción de magnitud...En lugar de esto, exijo lo siguiente: la aritmética debe desarrollarse a partir de sí misma... igualmente hemos de esforzarnos para definir los números irracionales exclusivamente mediante los números racionales. Sólo está en cuestión el cómo..."

“... Dedekind considera la introducción de los irracionales y los complejos como algo esencialmente problemático..."

“...Se procede entonces, a hacer una comparación de los números racionales con los puntos de una línea recta..."

“...en la línea recta $L$ hay infinitos puntos que no corresponden a ningún número racional... la recta $L$ es infinitamente más rica en individuos-punto que el domino $R$ de los números racionales en individuos-número..."

“...para su trabajo Dedekind considera la línea recta completa, sin huecos o sea continua...”

“...los números racionales no bastan para seguir aritméticamente todos los fenómenos de la recta, y en consecuencia se hace necesario crear nuevos números, de tal manera que el dominio numérico adquiera la misma completud o continuidad de la línea recta..."

“...el conjunto de los números racionales es denso pero no continuo (un conjunto es denso si está ordenado de tal manera que entre dos elementos cualquiera del mismo existe otro), y el problema es precisamente obtener un conjunto continuo, lo que presupone la definición satisfactoria de dicha propiedad..."

“...En el caso de las cortaduras es el orden del conjunto de los racionales -no su topologíalo que desempeña el papel fundamental..."

“... cada clase de equivalencia contiene infinitos elementos con lo que deja en claro que no tiene ningún reparo que hacer al infinito actual en matemáticas..."

Como ya se expuso, buscando tomar la esencia o las huellas que se vislumbran a través de estas frases se establecieron las siguientes categorías para el análisis de textos.

En las secciones previas al trabajo con números irracionales y reales, las categorías son:

Presentación de los conjuntos numéricos.

Justificación de los nuevos conjuntos numéricos.

Representación en la recta geométrica y/o recta numérica.

Relaciones de orden.

Otros elementos.

A su vez para analizar las secciones que presentan los números irracionales y reales, se establecieron categorías similares, incluyendo: 
Recta numérica y continuidad.

Infinito potencial - infinito actual.

Al igual que con el continuo numérico, para dar un sustento teórico al análisis de textos que se realizó, se recurrió a consultar recomendaciones básicas planteadas por algunos autores y se organizaron las preguntas, ¿Qué es un libro de texto?, ¿Qué características debe tener?, ¿Por qué son importantes los libros de texto para una política educativa?, ¿Por qué es importante el análisis de libros de texto?, ¿Para qué se analiza?, ¿Cómo se pueden analizar?, ¿Qué criterios se siguen para analizarlos?, ¿Cómo se clasifican los libros de texto seleccionados?, estableciendo respuestas para cada una de ellas. Se presenta dos.

\section{1 ¿QUÉ ES UN LIBRO DE TEXTO?}

"El texto escolar es un valioso instrumento educativo dado que materializa los programas curriculares, ayuda a la organización y administración del tiempo, presenta información verbal y gráfica estructurada pedagógicamente y propone actividades y ejercicios en sus páginas y fuera de ellas, que sirven para estimular y ayudar los procesos de pensamiento.

Un texto bien realizado puede contribuir a facilitar y a hacer más eficiente el trabajo del profesor y de los estudiantes y a mejorar la calidad de la educación". (Luis Bernardo Peña).

\section{2 ¿POR QUÉ ES IMPORTANTE EL ANÁLISIS DE LIBROS DE TEXTO?}

Como se afirma en el libro "Análisis de textos escolares de matemáticas", en los últimos años, en el ambiente educativo nacional, la indagación e investigación didáctica en torno a las matemáticas escolares han ganado en cobertura y profundidad. Este ambiente impone nuevas condiciones a la actividad docente de todos los profesionales en educación. Particularmente, constriñe la construcción de un conocimiento acerca de agentes y factores asociados a la actividad comunicativa de los saberes. Conocimiento que pretende dar cuenta de los rasgos característicos de los mismos.

El libro de texto de matemáticas, concebido como instrumento asociado a la comunicación de saberes matemáticos, es el instrumento mayoritariamente usado por los profesores. Especialmente el TIMSS (Tercer estudio internacional en ciencias y matemáticas), muestra que el texto es utilizado para decidir qué temas enseñar y cómo enseñarlos así como para determinar cuáles ejercicios y problemas solucionar. Esta posición privilegiada del texto, conduce indudablemente al reconocimiento de la necesidad de convertirlo en objeto de estudio didáctico, y, en consecuencia, de aprendizaje didáctico. En otras palabras, se hace imprescindible que los profesores construyamos un conocimiento altamente cualificado de los textos matemáticos, tal que potencie respuestas a preguntas sobre la calidad de los mismos, así como su mejor aprovechamiento -entre otras-.

En esta investigación ${ }^{1}$, para la selección de los textos se utilizó como criterio la identificación de los textos más usados en octavo grado en el área de matemáticas. La población encuestada estuvo conformada por 149 estudiantes que en el año 2001 estaban cursando primer semestre de licenciatura en matemáticas en la Universidad Pedagógica Nacional, y

\footnotetext{
${ }^{1}$ Elaborada por Vargas H. Jeannette, y Perilla R. Ciro.

Digitalizado por RED ACADEMICA
} 
primer semestre de Trabajo Social y Bacteriología y Laboratorio Clínico en la Universidad Colegio Mayor de Cundinamarca. Obteniendo los siguientes resultados:

\begin{tabular}{|l|l|l|l|}
\hline Texto & Autor & Editorial & Frecuencia \\
\hline Matemática 2000 & Mauricio Villegas Rodríguez & Voluntad S. A. 1991 & 18 \\
\hline $\begin{array}{l}\text { Matemática } \\
\text { constructiva 8 }\end{array}$ & $\begin{array}{l}\text { Gustavo Centeno, Hollman } \\
\text { Centeno, Nelson Jiménez, } \\
\text { Marco F. Robayo }\end{array}$ & $\begin{array}{l}\text { Libros y Libres S.A. } \\
1991 .\end{array}$ & 17 \\
\hline Álgebra elemental & Aurelio Baldor & $\begin{array}{l}\text { Cultural Colombiana. } \\
1971 .\end{array}$ & 102 \\
\hline Otros & & & 12 \\
\hline Total & & \multicolumn{2}{|l}{149} \\
\hline
\end{tabular}

Adicional a los tres textos con mayor frecuencia se seleccionó un texto de publicación reciente, elegido primordialmente por su presentación actualizada a los lineamientos curriculares del Ministerio de Educación Nacional.

\begin{tabular}{|l|l|lr|}
\hline Texto & Autor & Editorial & \\
\hline Nuevo & Leonor Camargo de Uribe, Gloria García de García, Grupo r Editorial \\
Alfa 8 & $\begin{array}{l}\text { Cecilia Leguizamón de Bernal, Carmen Samper de } \\
\text { Caicedo, Celly Serrano de Plazas. }\end{array}$ & $\begin{array}{l}\text { Norma Educativa. } \\
1999 .\end{array}$ \\
\hline
\end{tabular}

\section{CUADRO COMPARATIVO DE LAS OBSERVACIONES EN LAS SECCIONES PREVIAS A LA PRESENTACIÓN DE LOS NÚMEROS IRRACIONALES Y LOS NÚMEROS REALES}

A través de las observaciones establecidas para cada texto, ( cuadro no. 1), con una mirada que se denominó "horizontal", se comparan los textos teniendo en cuenta cada categoría de análisis y, se concluye:

El Texto Uno hace énfasis en la medición de magnitudes, el Texto Dos centra su mirada en establecer si cada conjunto numérico completa la recta, el Texto Tres hace referencia a aspectos históricos, mencionando la necesidad de medir magnitudes, el Texto Cuatro enfatiza en las aproximaciones y recurre a la observación de regularidades. 
CUADRO NO. 1 , COMPARATIVO DE LAS OBSERVACIONES EN LAS SECCIONES PREVIAS A LA PRESENTACIÓN DE LOS NÚMEROS IRRACIONALES Y LOS NÚMEROS REALES

\begin{tabular}{|c|c|c|c|c|}
\hline $\begin{array}{l}\text { TEXTO } \\
\text { CATEGORÍA } \\
\text { ANÁLISIS }\end{array}$ & TEXTO UNO & TEXTO DOS & TEXTO TRES & TEXTO CUATRO \\
\hline $\begin{array}{l}\text { PRESENTACIÓN DE } \\
\text { LOS CONJUNTOS } \\
\text { NUMÉRICOS }\end{array}$ & 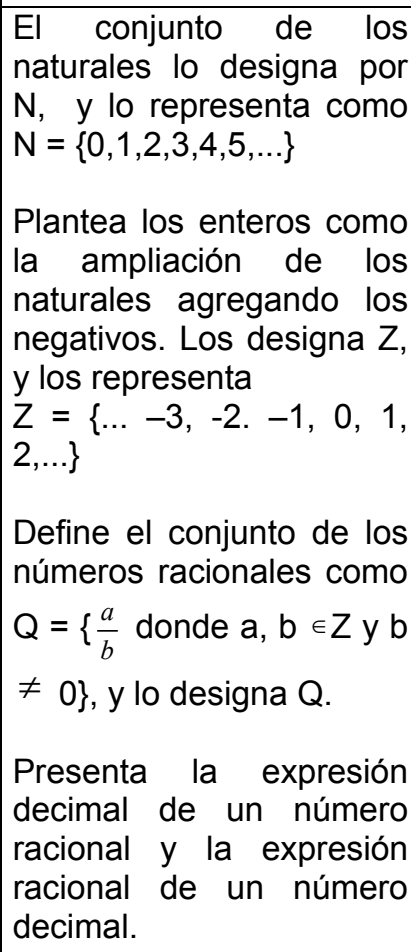 & $\begin{array}{l}\text { En el texto se presentan los conjuntos numéricos } \\
\mathrm{N}, \mathrm{Z}, \mathrm{Q} \text { afirmando que cada uno de ellos "tiene } \\
\text { infinitos elementos" } \\
\text { Retoman la idea de conjuntos coordinables para } \\
\text { definir los números naturales y con ellos formar } \\
\text { el conjunto de los números naturales. } \\
\text { Lo designan } \mathrm{N}, \text { y lo representan } \mathrm{N}= \\
\{0,1,2,3,4,5,6, \ldots, 100,101, \ldots, 1000, \ldots, 1.000 .000, \ldots\} \\
\begin{array}{l}\text { Se define el conjunto de los números enteros } Z \\
\text { como la unión de tres conjuntos, es decir }\end{array} \\
Z=Z^{+} \cup\{0\} \cup Z^{-} \\
\text {Aclaran que un número racional puede ser } \\
\text { positivo, cero o negativo y concluyen que } \\
Q=Q^{-} \cup\{0\} \cup Q^{+}\end{array}$ & $\begin{array}{l}\text { No se recurre a una } \\
\text { presentación } \\
\text { específica de los } \\
\text { conjuntos de } \\
\text { números naturales, } \\
\text { enteros y racionales. }\end{array}$ & $\begin{array}{l}\text { Inician con la expresión } \\
\text { decimal de un número } \\
\text { racional, no se hace } \\
\text { referencia específica de } \\
\text { los conjuntos de } \\
\text { números naturales, } \\
\text { enteros y racionales. } \\
\text { En el texto se presenta } \\
\text { el número racional } \\
\text { expresado de la forma } \\
\frac{a}{b} \text {, luego mediante su } \\
\text { expresión decimal, y su } \\
\text { equivalencia como suma } \\
\text { de fracciones decimales. }\end{array}$ \\
\hline $\begin{array}{l}\text { JUSTIFICACIÓN DE } \\
\text { NUEVOS } \\
\text { CONJUNTOS }\end{array}$ & $\begin{array}{l}\text { La ampliación de cada } \\
\text { conjunto numérico se } \\
\text { justifica por la existencia } \\
\text { de una(s) operación(es) } \\
\text { que no siempre es posible } \\
\text { en dicho conjunto. }\end{array}$ & $\begin{array}{l}\text { La ampliación de cada conjunto numérico se } \\
\text { justifica por el hecho de que hay una operación } \\
\text { que no siempre es posible en dicho conjunto. } \\
\text { Exponen, cada vez que abordan un conjunto, } \\
\text { ya sea N, Z, Q, que éste no completa la recta. }\end{array}$ & $\begin{array}{l}\text { Justifica la necesidad } \\
\text { de I } \\
\text { os nuevos conjuntos } \\
\text { numéricos, } \\
\text { adoptando un doble } \\
\text { criterio. Por un lado } \\
\text { un criterio histórico y } \\
\text { por otro, un criterio } \\
\text { intuitivo que ponga de } \\
\text { manifiesto las } \\
\text { necesidades que han }\end{array}$ & $\begin{array}{l}\text { En coherencia con la } \\
\text { observación descrita en } \\
\text { el punto uno, no se } \\
\text { puede hacer referencia a } \\
\text { la forma como se } \\
\text { justifica la ampliación de } \\
\text { los conjuntos numéricos }\end{array}$ \\
\hline
\end{tabular}




\begin{tabular}{|c|c|c|c|c|}
\hline NUMÉRICOS & & & $\begin{array}{lrr}\text { obligado } & \text { a } & \text { los } \\
\text { matemáticos } & \text { a } \\
\text { introducir } & \text { nuevos } \\
\text { entes numéricos. } \\
& & \\
\text { Desarrolla } & & \text { un } \\
\text { recuento } & \text { de } & \text { la } \\
\text { ampliación } & \text { de } & \text { los } \\
\text { conjuntos } & \text { numéricos, } \\
\text { haciendo referencia } \\
\text { únicamente } & \text { a } \\
\text { números positivos, en } \\
\text { congruencia con el } \\
\text { criterio } & \text { histórico } \\
\text { asumido en el texto }\end{array}$ & \\
\hline $\begin{array}{l}\text { JUSTIFICACIÓN DE } \\
\text { NUEVOS } \\
\text { CONJUNTOS } \\
\text { NUMÉRICOS }\end{array}$ & $\begin{array}{l}\text { Para la justificación del } \\
\text { conjunto de los números } \\
\text { racionales se presenta } \\
\text { una sección titulada "El } \\
\text { número y la longitud" y } \\
\text { anterior a la presentación } \\
\text { de los irracionales, dedica } \\
\text { la sección "Magnitudes } \\
\text { inconmensurables" en } \\
\text { donde recurre a la } \\
\text { historia, más exactamente } \\
\text { a las magnitudes. }\end{array}$ & $\begin{array}{l}\text { En el texto nombra la construcción de } \\
\text { conjuntos numéricos. Tanto bajo el título } \\
\text { "Construcción del Conjunto Q" como bajo la } \\
\text { frase “... una manera de llegar al conjunto de los } \\
\text { números enteros }(Z) \text {, a partir del conjunto } \mathrm{N} \text {. }\end{array}$ & \begin{tabular}{llrr} 
Justifica & & la \\
introducción & de & los \\
números & & & \\
fraccionarios & a & partir \\
de la & necesidad & de \\
medir & magnitudes \\
continuas & y & también \\
define & & números \\
fraccionario & & como \\
aquellos & que r & nos \\
permiten & expresar & el \\
cociente & de & una \\
división & inexacta. \\
Afirma & que & en \\
oposición & a & los \\
números & & \\
fraccionarios & se & tiene \\
los números & enteros, \\
los cuales & se & pueden \\
definir & como & los & que \\
expresan & el & cociente \\
de & una & \multicolumn{2}{c}{ división } \\
exacta & &
\end{tabular} & $\begin{array}{l}\text { Exponen un proceso } \\
\text { para construir los } \\
\text { racionales mediante } \\
\text { aproximaciones } \\
\text { decimales sucesivas }\end{array}$ \\
\hline
\end{tabular}




\begin{tabular}{|c|c|c|c|c|}
\hline $\begin{array}{l}\text { REPRESENTACIÓN } \\
\text { EN LA RECTA } \\
\text { GEOMÉTRICA Y/O } \\
\text { RECTA NUMÉRICA }\end{array}$ & $\begin{array}{l}\text { A medida que va } \\
\text { presentando los conjuntos } \\
\text { numéricos, enuncia la } \\
\text { representación de los } \\
\text { números en la recta } \\
\text { numérica. }\end{array}$ & $\begin{array}{l}\text { Ubican los números naturales, enteros y } \\
\text { racionales en una recta geométrica, } \\
\text { mencionando en cada caso, incluso en el } \\
\text { conjunto de los números racionales, que hay } \\
\text { puntos de la recta que no corresponden a ningún } \\
\text { número. }\end{array}$ & $\begin{array}{l}\text { Ubica los números } \\
\text { enteros en una recta } \\
\text { geométrica. } \\
\text { presentación de esta } \\
\text { ubicación se realiza } \\
\text { después de introducir } \\
\text { los números reales. }\end{array}$ & $\begin{array}{l}\text { Mediante } \\
\text { aproximaciones } \\
\text { decimales sucesivas, se } \\
\text { induce la ubicación del } \\
\text { número racional en la } \\
\text { recta numérica, a la vez } \\
\text { que se está exponiendo } \\
\text { un proceso para } \\
\text { construir los racionales, } \\
\text { proceso que en el caso } \\
\text { de los decimales } \\
\text { periódicos se repetirá } \\
\text { infinitas veces. }\end{array}$ \\
\hline $\begin{array}{l}\text { RELACIONES } \\
\text { ORDEN }\end{array}$ & $\begin{array}{l}\text { Enuncia que el conjunto } \\
\text { de los números naturales } \\
\text { constituye un sistema } \\
\text { ordenado. Dado un } \\
\text { número, existe el } \\
\text { siguiente y a excepción } \\
\text { del cero todos tiene } \\
\text { anterior, situación que no } \\
\text { se menciona, en el Texto, } \\
\text { en los números enteros y } \\
\text { no se excluye } \\
\text { explícitamente para los } \\
\text { números racionales. }\end{array}$ & $\begin{array}{l}\text { Presentan las propiedades de conjuntos de } \\
\text { números; en el conjunto de los números } \\
\text { racionales no existe el anterior ni el siguiente de } \\
\text { un racional dado. A diferencia de lo que sucede } \\
\text { en el conjunto de los enteros donde cada } \\
\text { número tiene un anterior y un siguiente. } \\
\text { Propiedad que también se cumple en el conjunto } \\
\text { de los números naturales, excepto el antecesor } \\
\text { para el cero. }\end{array}$ & $\begin{array}{l}\text { Identifica relaciones } \\
\text { entre los elementos } \\
\text { de cada conjunto } \\
\text { Presenta los axiomas } \\
\text { de orden solo cuando } \\
\text { abordan el conjunto } \\
\text { de los números } \\
\text { reales. }\end{array}$ & $\begin{array}{l}\text { No se presenta ninguna } \\
\text { exposición sobre el } \\
\text { significado de la relación } \\
\text { menor que, sin embargo } \\
\text { se recurre a ésta para la } \\
\text { construcción de los } \\
\text { encajonamientos. }\end{array}$ \\
\hline
\end{tabular}




\section{ANÁLISIS COMPARATIVO DE LA PRESENTACIÓN DEL CONTINUO NUMÉRICO EN CUATRO TEXTOS ESCOLARES DE OCTAVO GRADO}

Mediante una mirada que se denominó "horizontal", cuyo propósito es comparar los textos teniendo en cuenta las categorías de análisis utilizadas, algunas de las observaciones en las categorías son:

En la presentación de los conjuntos numéricos, se observa que mientras en el texto Uno se inclinan por presentar demostraciones por contraejemplo sobre números que no son racionales, en el Texto Dos nombran siempre la construcción del conjunto numérico, en el Texto Tres definen de los números racionales e irracionales, y en el Texto Cuatro se hace referencia a construcciones de los números irracionales.

Los Textos Uno y Dos presentan el conjunto de los números reales como la unión de los conjuntos racionales e irracionales, haciendo uso de la notación de conjuntos. En el texto Tres llaman reales al conjunto de los números racionales e irracionales y el Texto Cuatro sin llegar a nombrar conjuntos, se expresa; todos los números racionales e irracionales conforman los números reales.

Tanto en el texto Dos como en el texto Tres, consideramos que se presentan "errores", en el Texto Dos debido al uso de la notación $R=\{Q \cup \mathrm{I}\}$, y en el texto Tres por la definición cíclica: irracional como número real y conjunto de los reales al conjunto de los números racionales e irracionales.

En relación a la Justificación de nuevos conjuntos numéricos, el texto Dos se caracteriza por plantear la construcción de un conjunto numérico para completar la recta, los otros textos analizados recurren a la historia y a las magnitudes inconmensurables, es así como el texto uno dedica dos secciones denominadas "El número y la longitud" y "Magnitudes inconmensurables", antes de iniciar la presentación de los números irracionales, en el Texto cuatro se detienen a definir medidas conmensurables e inconmensurables. Enfatizamos en que, en éstos últimos textos se recurre a la historia y se encuentra un número que no es racional, con una mirada dependiente de la noción de magnitud.

En cuanto a la presencia del infinito potencial y el infinito actual se realizan diferentes miradas en las diversas secciones, pero se recurre primordialmente a analizar que los textos Uno, Dos y Tres, hacen referencia a conjuntos numéricos, con la existencia simultánea de todos sus elementos, asumen de esta manera el infinito actual, y, en el Texto cuatro se nombran números, esto permite considerar que se encuentra la presencia del infinito potencial. Sin embargo en todos los textos se encuentran tanto situaciones de infinito potencial como de infinito actual.

La observación efectuada a través de la categoría de análisis denominada Recta numérica y continuidad, nos permite analizar que tanto en el texto Uno y Cuatro exponen que cuando se representa en la recta los números reales la recta se llama recta real.

En el Texto Tres ubican en la recta los números enteros y no se hace referencia a la representación de irracionales en ella, en contraste con ello, la secuencia del Texto Dos permite observar que se parte de la recta geométrica y se va construyendo la relación entre puntos de la recta y números, mencionando en cada caso que el conjunto numérico no 
completa la recta, hasta llegar al conjunto de los reales de los cuales enuncian la propiedad de completar la recta o completez. En este último Texto y en el Texto Uno afirman que quedan infinitos puntos que no corresponden a números racionales y deben ser ocupados por números irracionales, a diferencia del Texto Cuatro en donde explican la manera de ubicar irracionales entre números racionales y concluyen que los números racionales al ser ubicados en la recta dejan "vacíos".

En los Textos Uno, Dos y Cuatro, se expone que a cada número real le corresponde un punto sobre la recta y a cada punto sobre la recta le corresponde un número real, asumiendo de manera implícita el continuo numérico. Vale la pena anotar que en el Texto Dos nombran la completez como la propiedad de los reales de completar la recta y en el Texto Cuatro se menciona que con los irracionales se estructura el continuo numérico y, por ende, los números reales. El Texto tres se caracteriza por enunciar el Axioma de continuidad.

Las relaciones de Orden se exponen en el Texto Tres mediante axiomas, y en los Textos Uno, Dos y Cuatro, se enuncia la relación "menor que", "mayor que", e "igual a", presentándolas como una extensión de la relación de orden de los racionales, exponiendo que dos números reales diferentes al ubicarlos en la recta numérica el que está a la izquierda es menor y el que está a la derecha es mayor.

$\begin{array}{lccccc}\text { A continuación se presenta el cuadro no.2, que permiten ilustrar } & \text { algunas de las } \\ \text { observaciones } & \text { en } & \text { estas } & \text { últimas } & \text { dos } & \text { categorías. }\end{array}$ 
CUADRO NO. 2. SÍNTESIS DEL ANÁLISIS A LA PRESENTACIÓN DEL CONTINUO NUMÉRICO EN LOS TEXTOS ESCOLARES

\begin{tabular}{|c|c|c|c|c|}
\hline $\begin{array}{l}\text { TEXTO } \\
\text { CATEGO } \\
\text { RÍA DE } \\
\text { ANÁLISIS }\end{array}$ & TEXTO UNO & TEXTO DOS & TEXTO TRES & TEXTO CUATRO \\
\hline $\begin{array}{l}\text { RECTA } \\
\text { NUMÉRIC } \\
\text { A Y Y } \\
\text { CONTINU } \\
\text { I-DAD }\end{array}$ & $\begin{array}{l}\text { Hemos aprendido a ubicar } \\
\text { cualquier número racional } \\
\text { en la recta numérica. Si } \\
\text { suponemos que se han } \\
\text { representado en la recta } \\
\text { numérica todos } r \text { los } \\
\text { números racionales, en } \\
\text { dicha recta quedan } \\
\text { infinitos puntos que no } \\
\text { corresponden a números } \\
\text { racionales y deben ser } \\
\text { ocupados por números } \\
\text { irracionales. } \\
\text { procedimiento geométrico } \\
\text { para representar un } \\
\text { número irracional se basa } \\
\text { en el teorema de } \\
\text { Pitágoras } \\
4 \\
\text { - La presentación es } \\
\text { nominal, no ha surgido del } \\
\text { entendimiento, tampoco } \\
\text { de la representación. No } \\
\text { se presenta ninguna } \\
\text { argumentación } \\
\text { sustente la anterior } \\
\text { afirmación, sencillamente } \\
\text { se presenta en texto. } \\
\text { Cabe preguntarse hasta } \\
\text { que punto al hacer esta }\end{array}$ & 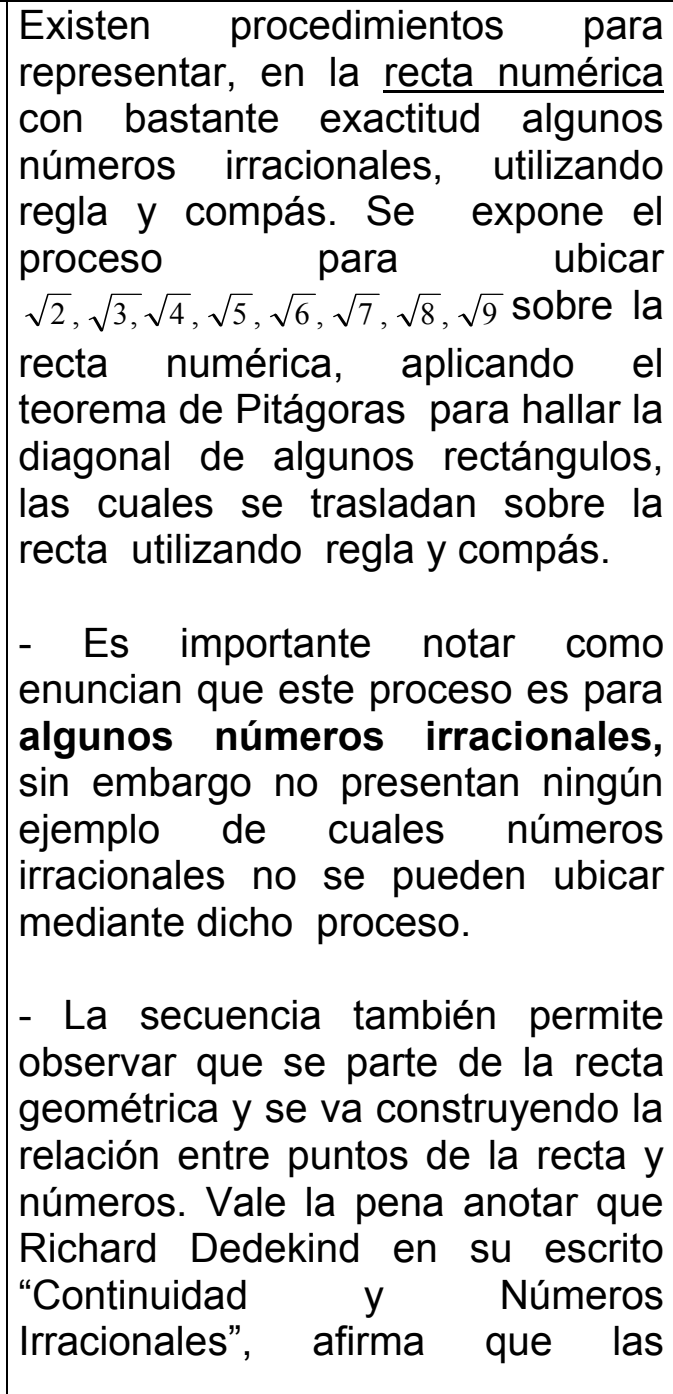 & 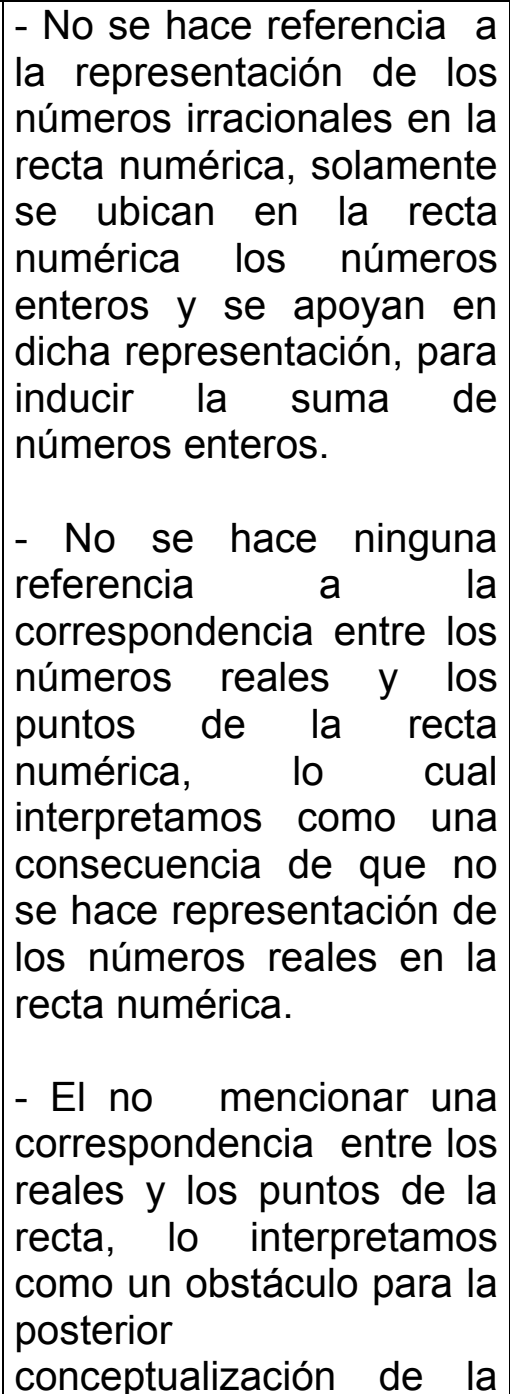 & $\begin{array}{l}\text { Al igual que los } \\
\text { números racionales, a } \\
\text { los irracionales les } \\
\text { corresponde un punto } \\
\text { en la recta numérica. } \\
\text { La manera más usual } \\
\text { de representarlos es } \\
\text { mediante } \\
\text { construcciones } \\
\text { geométricas y } \\
\text { significado del irracional } \\
\text { como medida. } \\
\text { Veamos la siguiente } \\
\text { construcción con regla } \\
\text { y compás } \\
\text { Dibujemos una recta, } \\
\text { marquemos el punto } 0 \\
\text { (llamado 0) y un } \\
\text { segmento de longitud } 1 \text {, } \\
\text {.. Así hemos colocado } \\
\text { el irracional } \sqrt{2} \text { en la } \\
\text { recta..., } \sqrt{2}, \sqrt{3} \text { se han } \\
\text { ubicado entre números } \\
\text { racionales, por tanto, } \\
\text { hay puntos en la recta } \\
\text { que no corresponden a } \\
\text { ningún número racional }\end{array}$ \\
\hline
\end{tabular}




\begin{tabular}{|c|c|c|c|c|}
\hline & $\begin{array}{l}\text { afirmación, sin ninguna } \\
\text { justificación sobre su } \\
\text { validez, se esta evadiendo } \\
\text { la argumentación sobre la } \\
\text { no continuidad de los } \\
\text { racionales. } \\
\text { Al representar los } \\
\text { números racionales en la } \\
\text { recta numérica, sabemos } \\
\text { que a cada número } \\
\text { racional le corresponde un } \\
\text { punto de la recta. Pero a } \\
\text { cada punto de la recta no } \\
\text { le corresponde } \\
\text { necesariamente r un } \\
\text { número racional porque } \\
\text { existen infinitos puntos } \\
\text { ocupados por números } \\
\text { irracionales }\end{array}$ & $\begin{array}{l}\text { propiedades de los números } \\
\text { racionales recuerdan las } \\
\text { relaciones de lugar recíprocas } \\
\text { entre los puntos de una línea. } \\
\text { A todo número racional se le puede } \\
\text { hacer corresponder un punto de la } \\
\text { recta, lo contrario no se cumple; } \\
\text { luego el conjunto Q, no completa la } \\
\text { recta. ¿Qué conjunto de números } \\
\text { se asignarán a los puntos de la } \\
\text { recta que no han sido asociados a } \\
\text { elementos de los conjuntos vistos? } \\
\text { - El conjunto Q no completa la } \\
\text { recta, afirmación que en palabras } \\
\text { de R. Dedekind “... la recta L es } \\
\text { infinitamente más rica en } \\
\text { individuos-punto que el dominio } R \\
\text { de los números racionales en } \\
\text { individuos-número...." es utilizada } \\
\text { en el documento de números } \\
\text { rracionales cuando argumenta } \\
\text { sobre la incompletud o } \\
\text { discontinuidad de los racionales. } \\
\text { En la construcción del conjunto de } \\
\text { los números irracionales (I), } \\
\text { observamos que en aquellos } \\
\text { puntos de la recta, donde no es } \\
\text { posible ubicar un número racional, } \\
\text { está un número irracional de tal } \\
\text { manera que estos dos conjuntos } \\
\text { completan la recta numérica } \\
\text { - En el texto no se enuncia la } \\
\text { continuidad de la recta, situación }\end{array}$ & 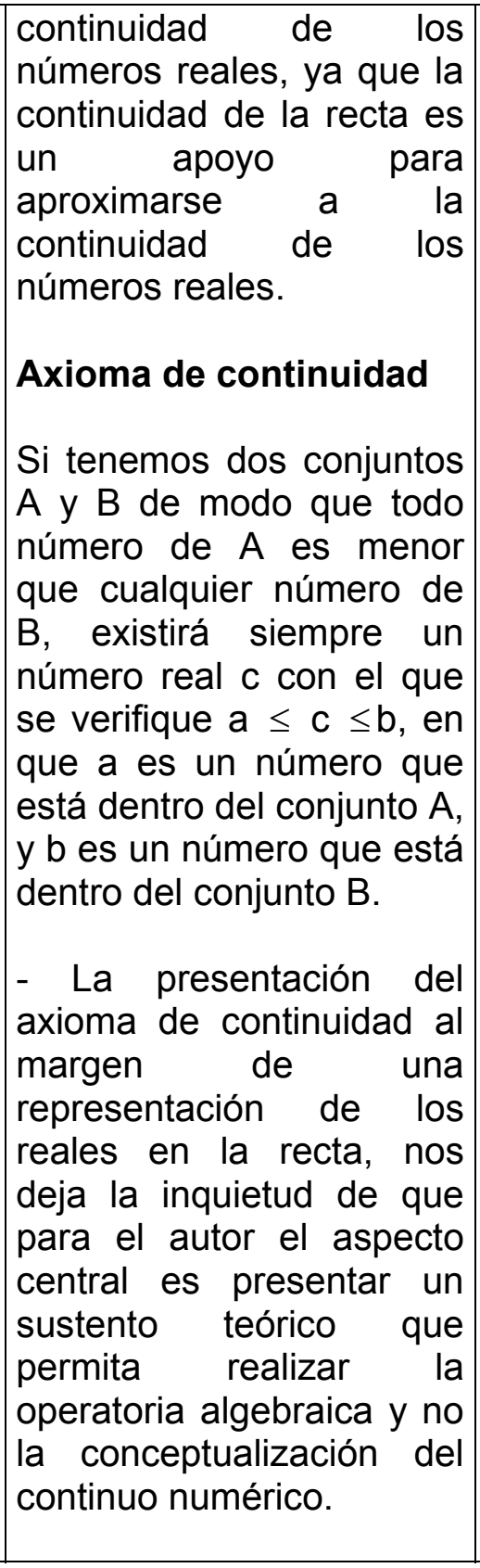 & $\begin{array}{l}\text { Es decir, los números } \\
\text { racionales al ubicarlos } \\
\text { en la recta dejan } \\
\text { "vacíos". } \\
\text { - Mediante este } \\
\text { procedimiento, ubicar } \\
\text { irracionales entre } \\
\text { números racionales, } \\
\text { concluyen que los } \\
\text { números racionales } \\
\text { dejan vacíos } \\
\text { A cada número real le } \\
\text { corresponde un punto } \\
\text { sobre la recta y a cada } \\
\text { punto sobre la recta le } \\
\text { corresponde un número } \\
\text { real. Cuando sobre la } \\
\text { recta se representan } \\
\text { los números reales la } \\
\text { recta se llama recta } \\
\text { real. } \\
\text { - Presenta una } \\
\text { secuencia, en las } \\
\text { unidades anteriores del } \\
\text { texto hacen mención a } \\
\text { la recta numérica y } \\
\text { cuando se representan } \\
\text { a los números reales la } \\
\text { llaman recta real. } \\
\text { Los números que están } \\
\text { a la derecha de } 0 \text { se } \\
\text { llaman reales positivos, }\end{array}$ \\
\hline
\end{tabular}




\begin{tabular}{|c|c|c|c|c|}
\hline $\begin{array}{l}\text { CONTINU } \\
\text { I-DAD }\end{array}$ & $\begin{array}{l}\text { recta real que tiene como } \\
\text { principal característica que } \\
\text { a cada punto le } \\
\text { corresponde número real } \\
\text { y a cada número real le } \\
\text { corresponde un punto. } \\
\text { - Se plantea que al ubicar } \\
\text { los números racionales e } \\
\text { irracionales en la recta } \\
\text { numérica existe una } \\
\text { correspondencia uno a } \\
\text { uno entre los puntos de la } \\
\text { recta y los números } \\
\text { reales, asumiendo de } \\
\text { manera implícita el } \\
\text { continuo numérico. }\end{array}$ & $\begin{array}{l}\text { que nos permite preguntar ¿con } \\
\text { qué fin se pretende completar la } \\
\text { recta?, al parecer no se está } \\
\text { buscando, o por lo menos no } \\
\text { explícitamente, que el 'nuevo' } \\
\text { conjunto numérico cumpla una } \\
\text { propiedad que ya tiene la recta; la } \\
\text { continuidad. Retomando a Richard } \\
\text { Dedekind "mediante la creación de } \\
\text { nuevos números tales que el } \\
\text { dominio numérico adquiera la } \\
\text { misma completud o, como } \\
\text { queremos decir igualmente, la } \\
\text { misma continuidad de la línea } \\
\text { recta. p. 83 } \\
\text { - Se hace explícita la existencia de } \\
\text { la biyección entre los números } \\
\text { reales y los puntos de la recta. "En } \\
\text { la recta numérica, cada punto } \\
\text { corresponde a un número real, y } \\
\text { cada número real corresponde a un } \\
\text { punto de la recta, es decir, se } \\
\text { puede establecer } \\
\text { correspondencia biunívoca entre } \\
\text { punto y número real". } \\
\text { - Han enunciado como propiedad } \\
\text { del conjunto Z y del conjunto Q el } \\
\text { no completar la recta, continúan la } \\
\text { presentación de los conjuntos con } \\
\text { la unión de Q e I, que tienen la } \\
\text { propiedad de completar la recta. } \\
\text { colocamos énfasis en que lo } \\
\text { anterior es expuesto en el texto } \\
\text { como una propiedad sencillamente }\end{array}$ & $\begin{array}{l}\text { - Este axioma de } \\
\text { continuidad, corresponde } \\
\text { al planteamiento hecho } \\
\text { por Dedekind. } \\
\text { - Es importante anotar que } \\
\text { Dedekind asumió la } \\
\text { continuidad de la recta } \\
\text { como un axioma con base } \\
\text { en el cual construyó un } \\
\text { conjunto numérico } \\
\text { continuo, el de los } \\
\text { números reales. Cabe } \\
\text { resaltar que en el texto se } \\
\text { presenta la continuidad de } \\
\text { los números reales como } \\
\text { un axioma. }\end{array}$ & 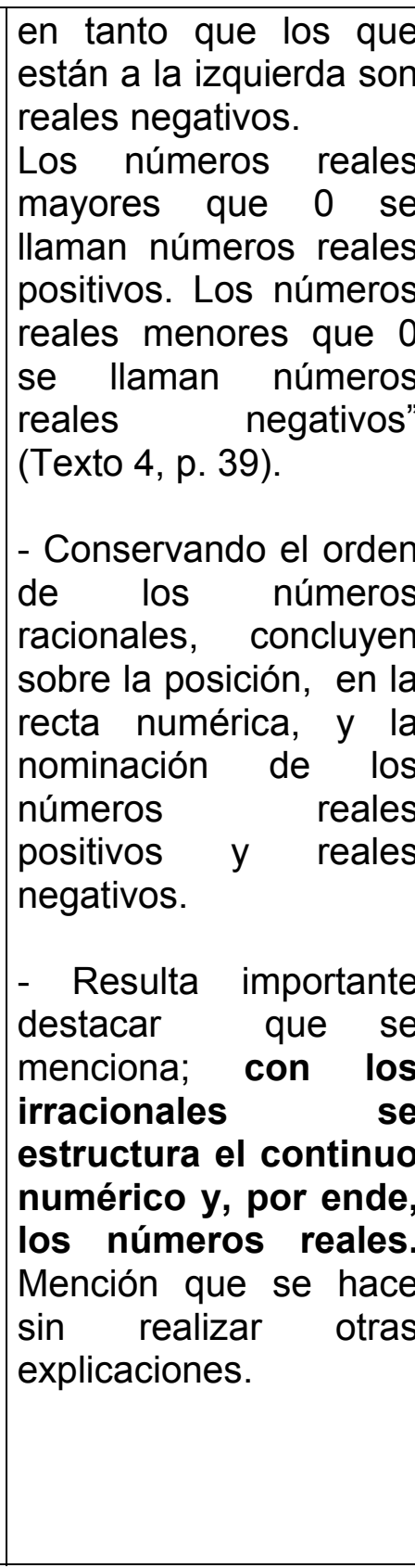 \\
\hline
\end{tabular}




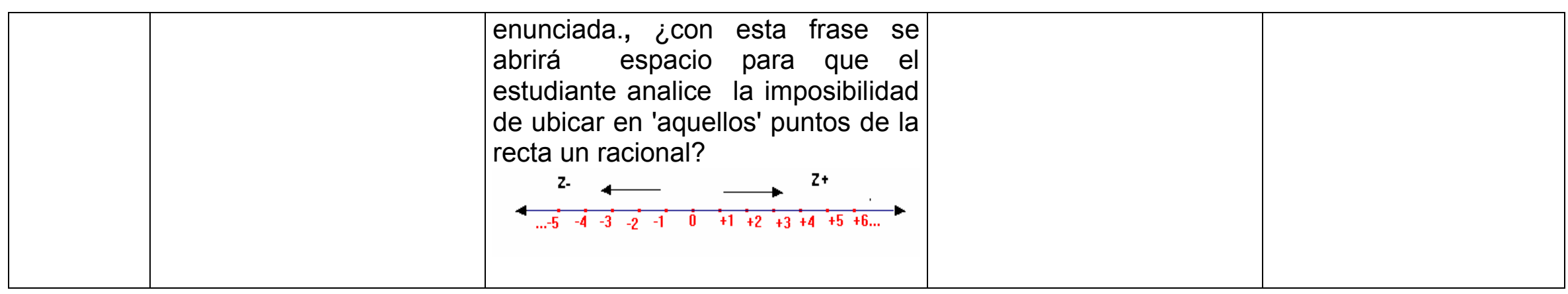




\begin{tabular}{|c|c|c|c|c|}
\hline $\begin{array}{l}\text { TEXTO } \\
\text { CATEGORÍA } \\
\text { DE ANÁLISIS }\end{array}$ & TEXTO UNO & TEXTO DOS & TEXTO TRES & TEXTO CUATRO \\
\hline $\begin{array}{l}\text { RELACIONES } \\
\text { DE ORDEN }\end{array}$ & $\begin{array}{l}\text { - La relación de orden se } \\
\text { presenta como una extensión de } \\
\text { la relación de orden de los } \\
\text { racionales. Parece como si el } \\
\text { criterio bajo el cual se enfoca el } \\
\text { texto, fuera la extensión de las } \\
\text { operaciones y la relación de } \\
\text { orden de los racionales a los } \\
\text { reales y no la construcción de } \\
\text { éstos a partir de los racionales y } \\
\text { su relación de orden. } \\
\text { - La forma de definir el orden en } \\
\text { los reales, se comparan cifras } \\
\text { decimales, no permite que se } \\
\text { establezca una diferencia entre el } \\
\text { tratamiento de los racionales y los } \\
\text { irracionales, por el contrario se } \\
\text { induce a que se opere con los } \\
\text { irracionales como si fueran } \\
\text { racionales, ya que tanto } \\
\text { decimales infinitos periódicos } \\
\text { como decimales infinitos no } \\
\text { periódicas se aproximan a } \\
\text { decimales finitos. } \\
\text { - Se asume la continuidad de la } \\
\text { recta numérica, la biyección entre } \\
\text { números reales y puntos de la } \\
\text { recta y se reafirma el convenio de }\end{array}$ & 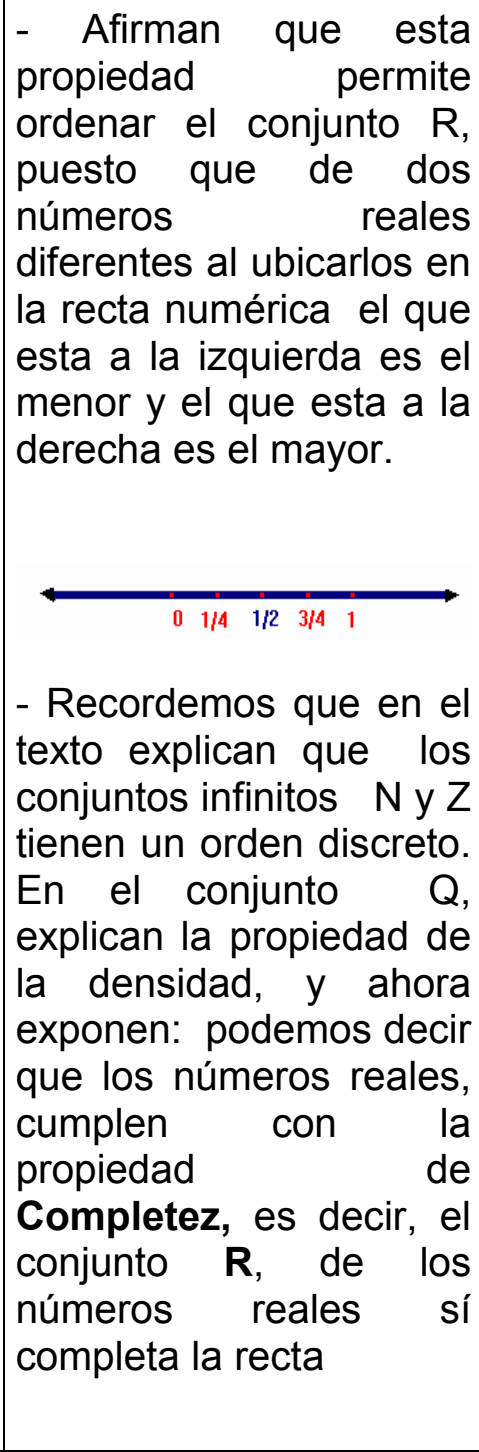 & 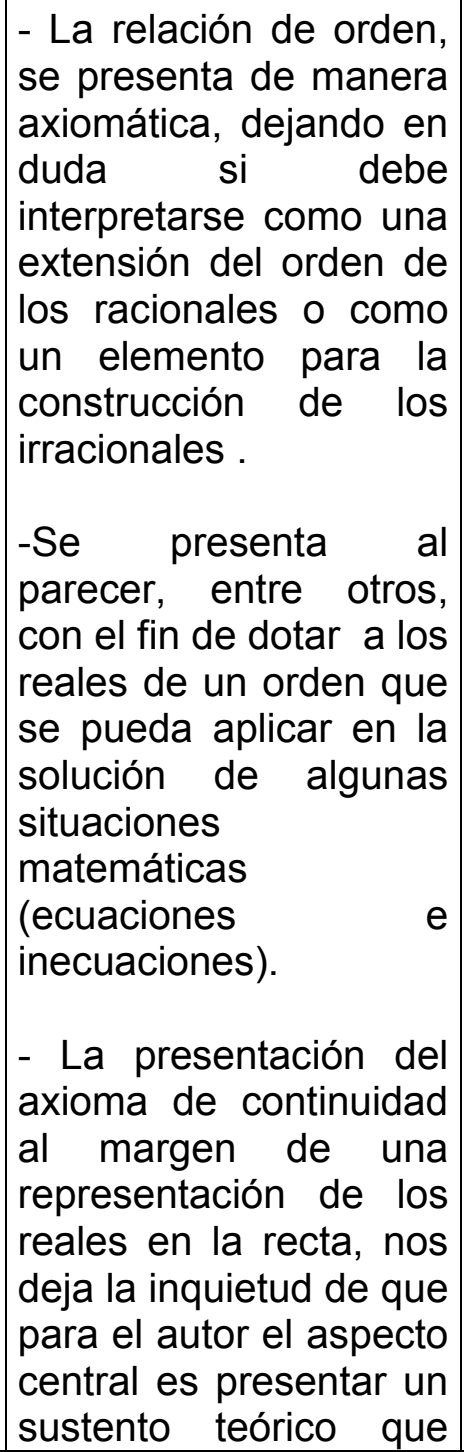 & $\begin{array}{l}\text { - Se recurre a la recta } \\
\text { numérica para asociar } \\
\text { el orden de los reales } \\
\text { con una representación } \\
\text { geométrica de los } \\
\text { mismos. } \\
\text { - A partir del orden de } \\
\text { los racionales } \\
\text { concluyen sobre el } \\
\text { orden de los reales }\end{array}$ \\
\hline
\end{tabular}




\begin{tabular}{|c|c|c|c|}
\hline & $\begin{array}{l}\text { que un punto ubicado a la } \\
\text { izquierda de otro, corresponde a } \\
\text { un número real menor que el } \\
\text { correspondiente al otro punto. } \\
\text { - Se recurre a la recta numérica } \\
\text { para asociar el orden de los } \\
\text { reales con una representación } \\
\text { geométrica de los mismos. }\end{array}$ & $\begin{array}{l}\text { - Surge el interrogante } \\
\text { ¿la frase hace pensar } \\
\text { que completez es } \\
\text { únicamente completar la } \\
\text { recta, pero sin que ello } \\
\text { implique algo más? }\end{array}$ & $\begin{array}{l}\text { permita realizar la } \\
\text { operatoria algebraica y } \\
\text { no la conceptualización } \\
\text { acerca del continuo } \\
\text { numérico. }\end{array}$ \\
\hline
\end{tabular}




\section{CONCLUSIONES}

Teniendo en cuenta que en esta investigación descriptiva, cualitativa e interpretativa, se elaboró un análisis de textos con el fin de responder a la pregunta, ¿De qué manera se presenta el continuo numérico en los textos escolares de matemáticas?, se establecen las siguientes conclusiones:

En el texto uno se asume de manera implícita el continuo numérico, al afirmar que en la recta numérica existe una correspondencia uno a uno entre los puntos de la recta y los números reales. Enfoca su trabajo a argumentar la existencia de números irracionales a partir de las magnitudes inconmensurables, a la vez que pretende formalizar la existencia de algunos irracionales mediante actividades prácticas de medición y demostraciones algebraicas.

El Texto dos pone de manifiesto que el problema central es construir un conjunto que complete la recta, exponen que los números reales, cumplen con la propiedad de Completez, es decir, el conjunto $R$, de los números reales sí completa la recta. Sin embargo al igual que todos los otros textos analizados, no hace explícita la continuidad de la recta geométrica.

En el Texto tres se privilegia la presentación axiomática del continuo numérico, mediante los axiomas de orden y el axioma de continuidad, asumiendo como aspecto central presentar un sustento teórico que permita realizar la operatoria algebraica y no la conceptualización acerca de los números reales.

Solamente en el Texto cuatro se realizan procesos de aproximación de los números irracionales, mediante encajonamientos, dejando abierto el camino hacia la conceptualización del continuo numérico.

\section{BIBLIOGRAFIA}

ARBELÁEZ, G., ARCE, J. GUACANEME, E. y SÁNCHEZ, G. 1999.Análisis de textos escolares de matemáticas. Universidad del Valle, Grupo de Educación Matemática.

BALDOR, A. 1971. Álgebra elemental. Bogotá: Cultural Colombiana.

CAMARGO, L., GARCíA de GARCÍA, G., SERRANO de PLAZAS, C. 1999. Nuevo Alfa 8. Bogotá: Grupo Editorial Norma Educativa.

CENTENO, G., CENTENO, H., JIMÉNEZ, N. y ROBAYO, M. F. 1991. Matemática constructiva 8. Bogotá, Libros y Libres S.A.

CHEVALLARD, Y. 1985. La transposición didáctica. Del saber sabio al saber enseñado. Grenoble: Editorial Savvages.

DEDEKIND, Richard.1998. ¿Qué son y para qué sirven los números?. Madrid: Alianza Editorial.

GARCÍA, G.; SERRANO, C. y ESPITIA, L. 1997 El concepto de función en textos escolares. Bogotá: Universidad Pedagógica Nacional - Colciencias. 
MONTERO, V. y TORRES, C. 1999. Reflexiones sobre las dificultades que conlleva la noción de infinito en el aprendizaje de la matemática. Revista Epsilon. No. 45. p. $357-364$

MORENO ARMELLA, L y WALDEGG, G. The conceptual evolution of actual mathematic infinity.

PERILLA, C. y VARGAS, J. 2003. El continuo Numérico. Un análisis de textos. Universidad Pedagógica Nacional. Tesis de Maestría. Bogotá.

ROMERO ALDABEJO, I. 1997.La introducción del número real en enseñanza secundaria: una experiencia de investigación-acción. Universidad de Granada.

RUSSELL, B. 1969.Definición de número. En: Newman, J. (comp.) Sigma, el mundo de la matemática. Vol. 4 (p. 116-118). Barcelona, España: Grijalbo.

SCHUBRING, G. 1992. Sobre la metodología de libros de texto históricos: Lacroix como autor de libros de texto. En: Mathesis. No. 8. p. 273-298. Bogotá.

VILLEGAS, M. Matemática 2000. 1997. Bogotá: Voluntad S.A. 